\title{
THE EFFECTS OF LINE-OF-SIGHT INTEGRATION ON MULTISTRAND CORONAL LOOP OSCILLATIONS
}

\author{
I. De Moortel and D. J. Pascoe \\ School of Mathematics and Statistics, University of St. Andrews, North Haugh, St. Andrews, Fife KY16 9SS, UK; ineke@mcs.st-and.ac.uk \\ Received 2011 October 13; accepted 2011 December 23; published 2012 January 23
}

\begin{abstract}
Observations have shown that transverse oscillations are present in a multitude of coronal structures. It is generally assumed that these oscillations are driven by (sub)surface footpoint motions. Using fully three-dimensional MHD simulations, we show that these footpoint perturbations generate propagating kink (Alfvénic) modes which couple very efficiently into (azimuthal) Alfvén waves. Using an ensemble of randomly distributed loops, driven by footpoint motions with random periods and directions, we compare the absolute energy in the numerical domain with the energy that is "visible" when integrating along the line of sight (LOS). We show that the kinetic energy derived from the LOS Doppler velocities is only a small fraction of the actual energy provided by the footpoint motions. Additionally, the superposition of loop structures along the LOS makes it nearly impossible to identify which structure the observed oscillations are actually associated with and could impact the identification of the mode of oscillation.
\end{abstract}

Key words: magnetohydrodynamics (MHD) - Sun: atmosphere - Sun: corona - Sun: oscillations - waves

Online-only material: color figures

\section{INTRODUCTION}

Historically, the dissipation of (MHD) waves and oscillations in the corona has been invoked as one way to provide (some of) the energy needed to account for the unexpectedly high coronal temperatures (several orders of magnitude higher than the solar surface; see reviews by, e.g., Walsh \& Ireland 2003; Klimchuk 2006; or Taroyan \& Erdélyi 2009). Although direct observations of waves and oscillations in the solar corona were absent for a long time, recent observations have revealed that a variety of small amplitude oscillations is present in almost all coronal structures. Alongside their possible role in coronal heating, MHD waves can be used as a seismological tool (Uchida 1970; Roberts et al. 1984) to provide estimates of local plasma parameters that are difficult to obtain by direct measurements (see reviews by, e.g., De Moortel 2005; Nakariakov \& Verwichte 2005; Erdélyi 2006; Banerjee et al. 2007; or De Moortel \& Nakariakov 2012). The recent confirmation of the ubiquitous presence of waves and oscillations in the solar atmosphere by, for example, Tomczyk et al. (2007) and McIntosh et al. (2011), is likely to substantially widen the possible application of coronal seismology and has reopened the debate on the role of wave heating in the solar atmosphere. The identification of the correct mode and the estimate of the energy budget will play a crucial role in both of these applications. Of particular interest in the coronal heating debate are the observations in the past few years of transverse oscillations propagating along the magnetic field in a variety of structures such as prominences (Okamoto et al. 2007), X-ray jets (Cirtain et al. 2007), spicules (De Pontieu et al. 2007; He et al. 2009a, 2009b), and coronal loops (Tomczyk et al. 2007; Tomczyk \& McIntosh 2009; McIntosh et al. 2011). Also, Jess et al. (2009) reported on the possible detection of torsional Alfvén waves in the lower solar atmosphere, with an energy budget that could be sufficient to account for the coronal heating requirements.

As the solar corona is optically thin, line-of-sight (LOS) integration means that in any given pixel of, for example, an EUV image, several coronal structures are likely to be present and this has to be taken into consideration when interpreting the observations. For example, the effect this LOS integration has on the appearance of coronal structures in EUV observations is discussed by DeForest (2007). Tomczyk et al. (2007) have shown that small amplitude transverse oscillations are likely to be present in a large number of (off-limb) coronal loops and Tomczyk \& McIntosh (2009) briefly discuss the effect of the LOS superposition and resolution on the estimated energy budget. Similarly, McIntosh et al. (2011) point out that the estimated energy budgets they report are likely to be lower limits, due to LOS superposition. Cooper et al. (2003) considered the effects of LOS integration on a single loop and demonstrated how this could potentially complicate the identification of observed oscillations. For example, the largely incompressible kink mode could still be associated with perturbations in the emission, depending on the angle between the loop and the LOS. Terradas et al. (2008) look at resonant absorption in a similar multistrand model but these authors focus on the actual resonant absorption process and do not consider the consequences of the LOS integration. In this paper, we investigate the effects of LOS superposition in a threedimensional (3D) numerical model of a multistrand structure. We make a qualitative, order of magnitude comparison between the "observed" energy budget (from Doppler shift oscillations), which will be greatly reduced due to the LOS superposition, with the energy budget actually present in the loop oscillations. We also address the issue of mode identification, especially in light of the recent observations of ubiquitous Doppler shift perturbations propagating in coronal loops.

The paper is organized as follows. The setup is described in Section 2, the results are analyzed in Section 3, and a discussion and conclusions are presented in Section 4.

\section{MODEL SETUP}

We consider a straight magnetic field in the (vertical) $z$-direction and choose the background plasma $\beta=0.01$ to be small, appropriate for the solar corona. Our density profile is composed of 10 loops, with random positions and density contrasts. The number of loops (10) was chosen on order of 


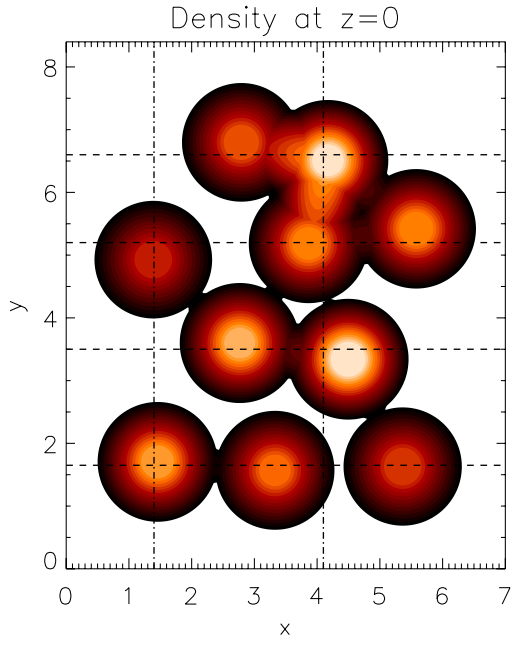

Figure 1. Contours of a horizontal cross section of the density (i.e., as a function of $x$ and $y$ ). The horizontal dashed and vertical dot-dashed lines represent the locations of density enhancements which appear to be loop structures when integrating over $x$ or $y$, respectively (see also Figure 2).

(A color version of this figure is available in the online journal.)

magnitude grounds, i.e., sufficiently high to actually allow for LOS superposition but small enough for the numerical resolution to be sufficiently high to resolve the mode coupling process. A high numerical resolution is needed to ensure that the phasemixed Alfvén waves (generated by the mode coupling process) do not reach scales below the grid resolution. Each individual loop is set up as in Pascoe et al. (2010); a vertical cylindrical tube, with a core region of constant density, surrounded by an inhomogeneous layer (referred to as the tube boundary or shell region). The density is $\rho_{0}$ in the core region and $\rho_{e}$ in the external region $(r>a)$. We choose density contrasts $\rho_{0} / \rho_{e}$ in the range 2.5-4, and an inhomogeneous layer thickness $l / a=0.75$. Figure 1 shows a contour plot of the cross section of the density at the base of our simulation $(z=0)$. The 10 loops with different density contrasts are clearly identifiable. Note that we are considering a coronal volume in our numerical simulations as the aim of this paper is to illustrate how estimates of the energy budget derived from observed coronal Doppler shift oscillations (Tomczyk et al. 2007; Tomczyk \& McIntosh 2009) are affected by the optically thin LOS superposition. Each loop has a temperature profile similar to that for density: $T_{0}$ in the core region, $T_{e}$ in the external region, and varying linearly in the inhomogeneous region. We choose a temperature contrast ratio of $T_{0} / T_{e}=1.1$ for all loops. In order to produce an equilibrium for our hot and dense loops, we vary the magnitude of the magnetic field strength so as to satisfy the condition of total pressure balance. Consequently, the magnetic field strength is up to $2 \%$ smaller inside the loops than outside. Inside the loops, the plasma $\beta$ rises up to 0.043 . All variables have been non-dimensionalized using typical values for the magnetic field strength $B_{0}$, the density $n$, and a typical lengthscale $L_{0}$.

\subsection{Driver}

The driving condition is applied to the lower $z$ boundary to simulate excitation by random footpoint motions. Our driver generates transverse velocity perturbations propagating along field lines. By placing our driver at the bottom of the coronal domain, we are trying to mimic observed Doppler shift oscillations. Although we are implicitly assuming these footpoint motions are related to solar surface motions, we do not consider the propagation of these oscillations through the atmosphere. Theoretically, our driver corresponds to moving the loop footpoint back and forth about its initial position, which is a very general perturbation that could be generated by a number of processes. The time dependence of our driver is based on a single period displacement of the loop axis as in Pascoe et al. (2010) and is applied from $t=0$ to $t=P_{0}$. This lower boundary driving generates a non-monochromatic propagating wavetrain along each loop, with the dominant period of oscillation in a Fourier spectrum being $P \approx \frac{2}{3} P_{0}$. Each loop has its own particular value of driving time $P_{0}$, randomly chosen in the range 13.5-16.5. A single pulse has been chosen rather than (possibly more realistic) continuous or quasi-periodic driving to keep the perturbations and their associated energy budgets tractable. Although continuous driving would be a more accurate reflection of solar surface motions, the effect of the optically thin LOS integration on the estimated energy budget and the mode identification will be essentially the same. Indeed, the single pulse used in this study to illustrate the LOS effects could be seen as a single wavelength (or driving period) of a (harmonic) wavetrain or quasi-periodic wavepacket and the mode coupling process has more or less the same effect on a single wave pulse as it does on a harmonic wavetrain. The spatial dependence of the driver is based on a two-dimensional dipole (see Pascoe et al. 2010, 2011) and each loop has a direction of oscillation randomly chosen in the $x-y$-plane. The maximum amplitude of the transverse velocities at the lower boundary is chosen to be small $\left(u_{0} / C_{A e}=0.01\right)$ to approximate a linear regime.

The simulations are performed using the MHD code LARE3D (Arber et al. 2001) with $400 \times 400 \times 300$ grid points for a numerical domain of $70 \times 84 \times 500 \mathrm{Mm}$. The boundary conditions are periodic in the $x$ - and $y$-directions, and are placed sufficiently far away to not affect the results. Initially the lower $z$ boundary is driven, but after our driving phase $\left(t \leqslant P_{0}\right)$ the driver is turned off and the $z$ boundaries also become periodic. This avoids the need for a large domain in the field-aligned direction by allowing the wavetrain to propagate out of the top of our domain and re-enter at the lower boundary. We remind the reader that, throughout this manuscript, non-dimensional values of all parameters are used. Dimensional quantities can be obtained by assuming a normalizing value of the magnetic field $B_{0}$, a typical lengthscale $L_{0}$, and number density $\rho_{0}$ (see Arber et al. 2001). For example, using a value of $B_{0}=10 \mathrm{G}$, $L_{0}=10 \mathrm{Mm}$, and $\rho_{0}=10^{15} \mathrm{~m}^{-3}$ gives a timescale of $t_{0}=14.5$ s. Hence with this normalization the periods of 13.5-16.5 would correspond to 200-240 s.

\section{EFFECTS OF LINE-OF-SIGHT INTEGRATION}

\subsection{Density (Intensity) Structures}

Ten different flux tubes are present in the numerical domain and these 10 individual structures are clearly identifiable in the horizontal cross section shown in Figure 1. However, superposition along the LOS effectively means summing some of these structures together. To simulate optically thin intensity, the top panel of Figure 2 shows a cross section of the density squared, integrated along the $x$-direction (and subsequently divided by the appropriate number of gridpoints to avoid artificially high values of density). As indicated by the vertical dashed lines, there are four clearly distinct maxima and hence when the $x$-direction coincides with the LOS (i.e., an observer is placed further along the $x$-axis), only 4 loops would be visible, rather than the 10 loops which are actually present in 

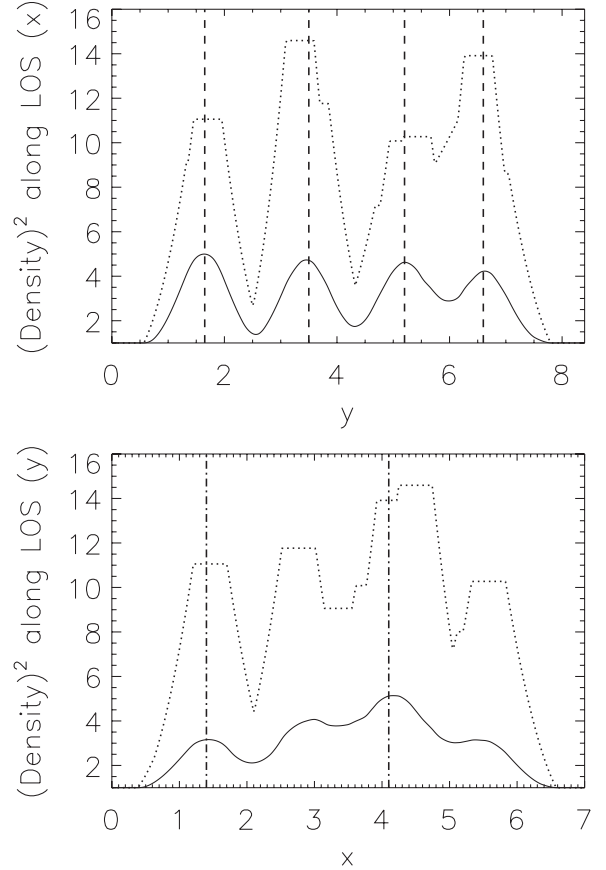

Figure 2. Cross section of the average density squared (i.e., divided by the number of gridpoints) integrated along the $x$-direction (top panel) and $y$-direction (bottom panel). The vertical dashed (dot-dashed) lines identify the maxima and correspond to the horizontal dashed (vertical dot-dashed) lines in Figure 1. The dotted lines correspond to the maximum value of the density squared in the $3 \mathrm{D}$ numerical domain at each $y(x)$.

the 3D domain. Similar integration along the $y$-direction reduces the number of "visible" loops to two as indicated by the dotdashed lines in the bottom panel of Figure 2. The positions of the maxima in the LOS integrated intensities (density squared) are also presented in Figure 1 as horizontal dashed lines (corresponding to the $x$-LOS integration) and vertical dotdashed lines ( $y$-LOS) to illustrate how the summations have reduced the number of visible structures to four and two, respectively. Generally, the locations of the peaks in the averaged densities do not correspond exactly to the locations of the center of the loop structures present in the 3D domain. In no case does an LOS integrated "loop" actually correspond to a single loop in the 3D domain. Also shown in Figure 2 by the dotted lines is the maximum value of the density squared in the $3 \mathrm{D}$ numerical domain at each $y(x)$. Comparing the dotted lines, which reflect the actual intensity contrast $\left(\rho_{0} / \rho_{e}\right)^{2}$ present in the domain, with the solid lines, we notice that the LOS summation does not only confuse the location of the actual structures but substantially reduces the intensity contrasts. This is especially noticeable for the $y$-LOS, where the averaged LOS intensity appears almost uniform. Hence, a region which appears nearly uniform, or with very low intensity contrast, could actually be a bundle of closely spaced loops with substantial density contrasts, implying that seismologically inferred density contrasts (from observed damping lengths) might not correspond well with the density (intensity) contrast visible in the LOS integrated observations.

\subsection{LOS Doppler Velocities}

As described in Section 2, each of the 10 structures is now subjected to an oscillatory displacement at the base of our numerical domain. For a single loop, the resulting mode coupling is described comprehensively by Pascoe et al. (2010, 2011); such perturbations travel along the loop and efficiently couple to an azimuthal Alfvén wave in the tube boundaries. This mode coupling process has been investigated in great detail recently (Pascoe et al. 2010, 2011; Terradas et al. 2010; Verth et al. 2010; Soler et al. 2011a, 2011b). What is important for our current investigation is that the damping length of the central, transverse displacement is (mainly) governed by the period of the footpoint driver, the density contrast $\rho_{0} / \rho_{e}$, and the relative thickness of the tube boundary (Hollweg \& Yang 1988; Goossens et al. 1992; Ruderman \& Roberts 2002; Pascoe et al. 2010; Terradas et al. 2010). The width of the shell region is the same for all 10 loops but the driving periods and density contrasts are different for each of the loop structures. Hence, they will each have different damping lengths, implying that at any given height, a mixture of transverse displacements and/or azimuthal Alfvén waves, at different phases of oscillation, will be present: at low height, the velocity perturbations will be dominated by the transverse (kink) modes whereas at large heights, only the azimuthal Alfvén waves will remain. At intermediate heights, both modes will be present and hence an integration along a given LOS will capture velocity perturbations that are a mixture of the transverse displacements and azimuthal Alfvén modes, as both are linear combinations of $v_{x}$ and $v_{y}$. To illustrate this, Figure 3 shows the horizontal velocity vectors in two different horizontal planes, at $z=1$ (top) and $z=165$ (bottom) at $t=15$ and $t=100$, respectively. It is clear that the lower height (top panel) is dominated by the randomly directed, transverse displacements induced by the bottom boundary driver, whereas the higher plane (bottom panel) is dominated by the azimuthal perturbations in the loop boundaries. In other words, the energy has moved from the cores of the loops to the outer shell regions due to mode coupling of the driven kink modes to the (azimuthal) Alfvén modes.

The LOS summation will make it difficult to uniquely identify individual structures (irrespective of the additional question of whether current observations actually resolve such structures) and hence it is already clear that it will be non-trivial to actually associate observed Doppler velocities with a given structure in the LOS. Additionally, as illustrated below, loop structures and their associated oscillations could line up in such a way as to make the (azimuthal $m=1$ ) Alfvén waves in the tube boundaries appear as larger bulk motions in the LOS integrated density structures.

In Figure 4, the LOS velocities that would be observed either along the $x$ - or $y$-axis are shown. These LOS velocities have been calculated by averaging the density-squared weighted velocity components along the LOS, i.e., $\left\langle\rho^{2} v_{x, y}\right\rangle /\left\langle\rho^{2}\right\rangle$ for the $x, y$ LOS and where $\langle\cdots\rangle$ represents the average along the appropriate LOS. Zacharias et al. (2011) have shown (see their Figure 4) that this intensity-weighted velocity accurately reflects Doppler shifts of optically thin emission lines. The first two panels of Figure 4 correspond to the $x$-axis being the LOS and the colored contours represent $v_{x}$, integrated over $x$. Observationally, these perturbations would correspond to periodic Doppler velocities traveling along magnetic structures (loops). As oppositely directed perturbations in the LOS will cancel each other out, the resulting Doppler velocities will be much smaller than the actual values in the numerical domain. For the $x$-LOS, the resulting Doppler velocities appear to line up reasonably well with the apparent density structures, even though the maxima in the velocities do not always correspond to the actual maxima in the density structure. However, for the $y$-LOS (right panels), there is a strong velocity perturbation which is not actually associated with a loop structure (the central 

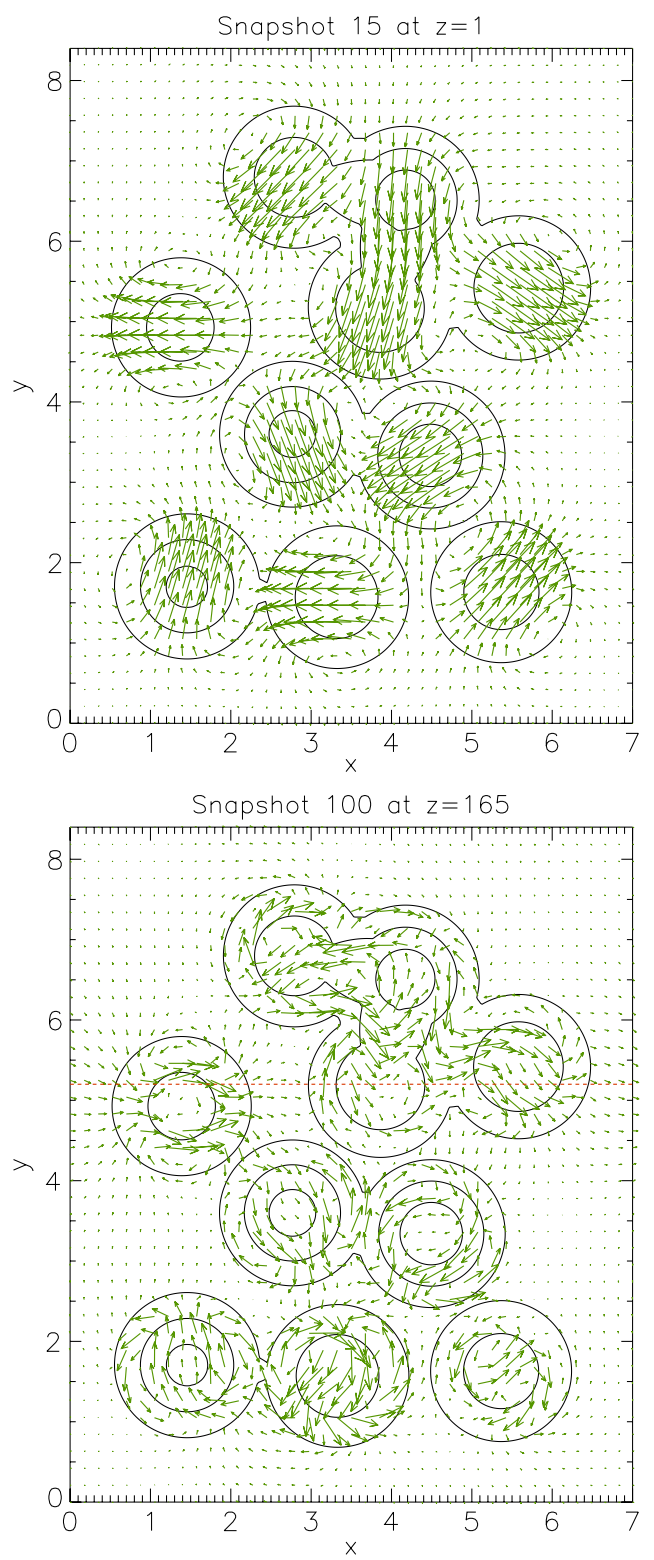

Figure 3. Velocity vectors in the horizontal plane at $t=15(z=1)$ and $t=100(z=165)$. The line contours correspond to the density and outline the loop structures. Note that the vectors in both panels have not been scaled to a common maximum.

(A color version of this figure is available in the online journal.)

oscillations), but would appear to be occurring in the wing of a wide structure (see the bottom panel of Figure 2). At early times, both the $x$ and $y$ integrated velocities would mainly correspond to the (bulk) transverse loop motions induced by the footpoint driver. At later times (i.e., higher heights), the remaining velocity perturbations are much weaker and mostly situated in the tube boundaries, as illustrated in the bottom panel of Figure 3. However, when integrated along the LOS, the azimuthal motions in the shell regions of neighboring loops could appear as bulk motions of the central part of an integrated loop. This has occurred in the $x$-LOS where, at $y=5.2$, an apparent bulk motion is still visible at later times. However, closer inspection of the planar velocities at, e.g., $z=27.5$ reveals that what appears to be a bulk Doppler velocity along the LOS loop centered on $y=5.2$ is mainly composed of the azimuthal Alfvén waves in the boundary of the flux tube centered on $[x=1.4, y=4.9]$ (see horizontal dashed line in the bottom panel of Figure 3).

\subsection{Energy Budget}

Finally, we focus on the effect of the LOS integration on the (apparent) energy budget. Figure 5 shows the evolution of the horizontal kinetic energy, i.e., only the horizontal velocity components $v_{x}$ and $v_{y}$ have been taken into account. The solid line corresponds to this horizontal kinetic energy integrated over the full 3D numerical domain at each timestep. The dashed and dot-dashed lines represent the LOS kinetic energies, obtained by using the LOS intensity-weighted velocity components (the summed $\left.\left\langle\rho^{2} v_{x, y}\right\rangle /\left\langle\rho^{2}\right\rangle\right)$, multiplied by the appropriate average density (see Figure 2). It is clear that using the observed Doppler velocity along either LOS combined with the averaged density will underestimate the energy that is actually present in the 3D domain, by as much as an order of magnitude. In the $y$ direction, the kinetic energy is never more than $40 \%$ of the total kinetic energy present in the 3D domain, whereas the $x$-LOS only gives about $10 \%$. In addition, the transverse driving of the loop footpoints will also result in magnetic perturbations and hence magnetic energy. The magnetic energy present in the numerical domain is of the same order as the kinetic energy, implying that the total energy contained in the transverse perturbations is double of the kinetic energy alone (see the dotted line in Figure 5). Hence, the (kinetic) energies estimated from the LOS Doppler velocities would only capture 5\%-20\% of the energy actually present in the $3 \mathrm{D}$ domain. It is clear that energy estimates based on observed Doppler velocities could underestimate the actual energy present in footpointdriven (transverse) loop oscillations by at least one, possibly two if more loops are considered, orders of magnitude. It is important to keep in mind that the LOS integrated energy cannot easily be associated with the different wave modes (either the transverse, propagating kink waves or the azimuthal Alfvén waves generated by the mode coupling). Indeed as shown by Pascoe et al. (2010) (see their Figure 9), there is a gradual energy transfer for each loop from the transverse mode in the core to the Alfvén waves in the shell region. Hence, apart from the very lowest heights in our numerical domain, where almost all the energy will be in the boundary-driven kink mode, the LOS integrated energy will contain a mixture of the kink mode and Alfvén wave contributions until all the energy from the kink mode in the core has been transferred to the azimuthal Alfvén waves in the shell regions.

\section{DISCUSSION AND CONCLUSIONS}

Even though their interpretation has been under debate, it has become clear that propagating, transverse oscillations are present in most observed coronal structures. Having successfully been modeled as coupled kink and (azimuthal) Alfvén waves (e.g., Pascoe et al. 2010; or Terradas et al. 2010), these "Alfvénic" waves appear to contain sufficient energy to account for the quiet-Sun and solar wind requirements (McIntosh et al. 2011). As they appear to be present all the time, in large numbers, these transverse oscillations also have the potential to be a powerful seismological tool. Indeed, with a large number of observed examples, statistical studies of the seismologically derived properties become possible, which will allow a more thorough evaluation of the properties so far derived from a relatively small sample of observations. 

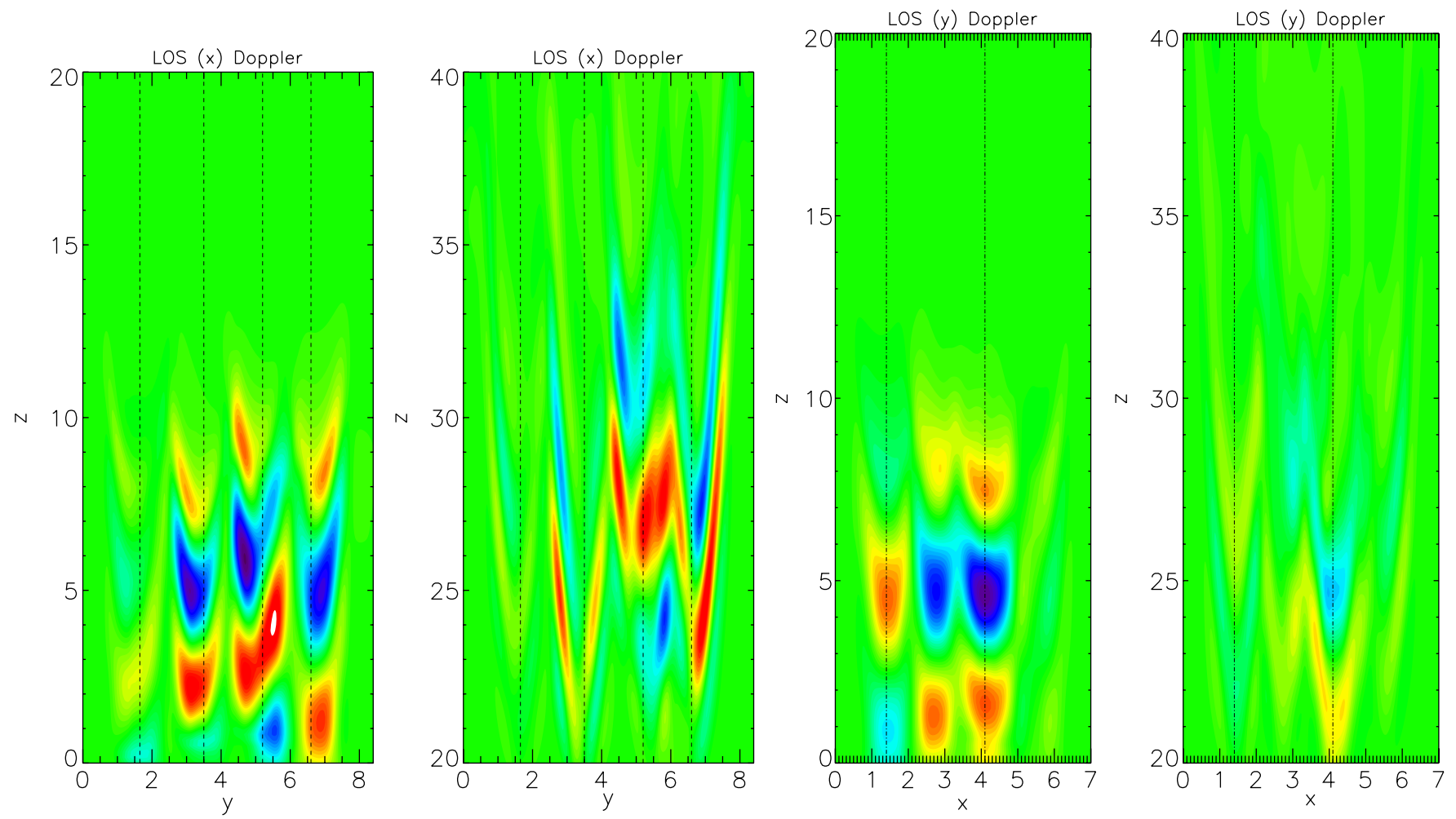

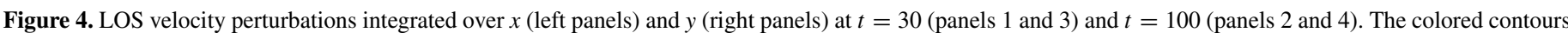

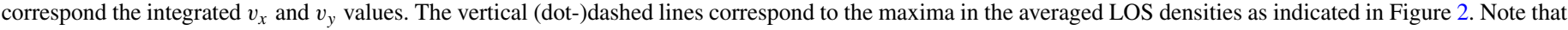
the maxima in the panels at later times have been scaled to half the value of the maxima at earlier times to make the perturbations visible.

(A color version of this figure is available in the online journal.)

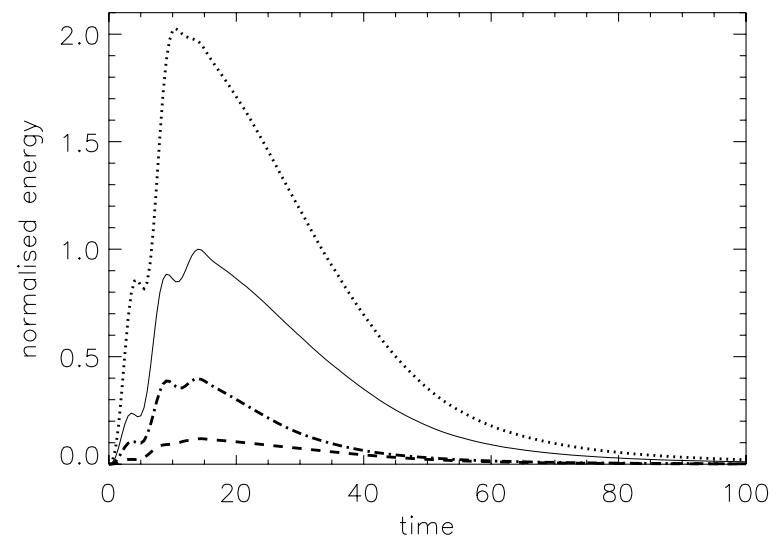

Figure 5. Normalized energy as a function of numerical timesteps. The solid line correspond to the kinetic energy in the 3D numerical domain whereas the dotted line corresponds to the total energy, i.e., kinetic plus magnetic energy, in the 3D numerical domain. The dashed and dot-dashed lines correspond to the kinetic energy resulting from the $x$ - and $y$-LOS integrated velocities, respectively.

Using a simple model of 10 randomly distributed loops, driven by randomly directed footpoint displacements, our simulations are intended to demonstrate how the LOS integration of optically thin emission lines could affect the identification of observed oscillations as well as the energy budgets. As several loop structures in the LOS are likely to be superimposed, associating observed Doppler velocities (i.e., the components of the velocity perturbations which are directed (anti-)parallel to the LOS) with specific structures is non-trivial. Additionally, the dependence of the damping length of the driven kink mode (through mode coupling) on the density contrast, driving period, and loop (transverse) structure means that the perturbations will have different damping lengths in different loops and hence integrated velocities are likely to contain a mixture of (bulk) transverse velocities from the cores of the loops and (azimuthal) Alfvén perturbations from the loop boundaries. The interpretation of these oscillations as "Alfvénic" appears attractive not only from a theoretical point of view (to reflect the generic coupled character of the mode) but also from an observational point of view (as the observed Doppler shift oscillations are likely to contain a mixture of kink and azimuthal Alfvén waves at any given height). In this study, estimating the energy based only on the integrated LOS intensity-weighted velocity components (representing Doppler shifts of optically thin emission lines) underestimates the energy actually present in the 3D domain, by as much as an order of magnitude (and possibly two orders of magnitude if the magnetic energy associated with the perturbations is included in the full 3D energy budget). In any case, there will be more energy available to couple to the Alfvén waves in the loop boundaries and ultimately, through the process of phase mixing, to heat the coronal loops than LOS estimates based on observed coronal Doppler shift oscillations might suggest.

Using only 10 loops (or loop threads) in our numerical domain, we have demonstrated some of the effects the LOS integration, inevitable in an optically thin environment, could have on the identification of structures and their associated oscillations, as well as the energy budget contained in these perturbations. The magnitude of these effects however is very dependent on the setup, in particular on the period and structure of the boundary driver, the number of loops in the LOS, and the density contrast of the individual loops with the background coronal environment. In the present study, we considered a relatively small range of density contrasts and driving periods, chosen specifically to mimic the COMP observations (Tomczyk 
et al. 2007; Tomczyk \& McIntosh 2009). The damping lengths of the propagating, transverse perturbations (or in other words the rate at which energy will be transferred from the transverse oscillations in the core to the Alfvén waves in the shell regions of the loop) strongly depend on the period and the density contrast. Higher frequencies and stronger density contrasts will lead to shorter damping lengths. Hence, considering a wider range of these parameters will result in a wider range of damping lengths present in the domain. This would imply that, at any given height, an even greater mixture of the kink and azimuthal Alfvén waves would be present in the LOS. The structure of the driver will also play a crucial role. In our setup, we considered a driver with a spatial scale equal to the cross section of the individual loop strands. As the direction of the transverse driving for each loop was chosen randomly, this leads to significant cancellation of the velocity perturbations along the LOS. If on the other hand all loops are driven in the same way, i.e., the spatial scale of the driver is considerably larger than the cross section of the loop strands, then all the loops will oscillate in phase and there will be no cancellation of velocity perturbations along the LOS. A final important factor is of course the number of loops present along the LOS. This will depend both on the location of the loop oscillations and on the spatial resolution of the observations. For off-limb loop oscillations and instruments with lower spatial resolution (COMP) the effects will be biggest as there is likely to be a large number of loops present along the LOS and within a single pixel. For on-disk observations of, e.g., a quiet-Sun region, the effects are likely to be much smaller. This is consistent with the findings of Tomczyk \& McIntosh (2009) and McIntosh et al. (2011): the energy flux contained in off-limb COMP (lower spatial resolution) observations of quasi-periodic Doppler shift oscillations was found to be several orders of magnitude smaller than similar quiet-Sun observations of transverse perturbations made by $S D O /$ AIA.

In summary, the effects of the LOS integration depend on a large number of factors. It is likely that, in reality, far more than 10 loop strands will be present in the LOS, especially in active regions and/or off-limb observations and additional effects such as curvature would also have to be taken into account. Indeed, more complicated (curved) structures will further enhance the effects we have described. Our results also imply that properties derived through coronal seismology are likely to reflect averaged properties of an ensemble of coronal loops.

I.D.M. acknowledges support of a Royal Society University Research Fellowship. D.J.P. acknowledges financial support from STFC. Computational time on the Linux clusters in St. Andrews (STFC and SRIF funded) is gratefully acknowledged. The authors thank Drs. H. Peter and S. McIntosh for valuable discussions.

\section{REFERENCES}

Arber, T. D., Longbottom, A. W., Gerrard, C. L., \& Milne, A. M. 2001, J. Comput. Phys., 171, 151

Banerjee, D., Erdélyi, R., Oliver, R., \& O’Shea, E. 2007, Sol. Phys., 246, 3

Cirtain, J. W., Golub, L., Lundquist, L., et al. 2007, Science, 318, 1580

Cooper, F. C., Nakariakov, V. M., \& Tsiklauri, D. 2003, A\&A, 397, 765

DeForest, C. E. 2007, ApJ, 661, 532

De Moortel, I. 2005, Phil. Trans. R. Soc. A, 363, 2743

De Moortel, I., \& Nakariakov, V. M. 2012, Phil. Trans. R. Soc. A, in press

De Pontieu, B., McIntosh, S. W., Carlsson, M., et al. 2007, Science, 318, 1574

Erdélyi, R. 2006, Phil. Trans. R. Soc. A, 364, 289

Goossens, M., Hollweg, J. V., \& Sakurai, T. 1992, Sol. Phys., 138, 233

He, J.-S., Marsch, E., Tu, C.-Y., \& Tian, H. 2009a, ApJ, 705, L217

He, J.-S., Tu, C.-Y., Marsch, E., et al. 2009b, A\&A, 497, 525

Hollweg, J. V., \& Yang, G. 1988, J. Geophys. Res., 93, 5423

Jess, D. B., Mathioudakis, M., Erdélyi, R., et al. 2009, Science, 323, 1582

Klimchuk, J. A. 2006, Sol. Phys., 234, 41

McIntosh, S. W., De Pontieu, B., Carlsson, M., et al. 2011, Nature, 475, 477

Nakariakov, V. M., \& Verwichte, E. 2005, Living Rev. Solar Phys., 2, 3

Okamoto, T. J., Tsuneta, S., Berger, T. E., et al. 2007, Science, 318, 1577

Pascoe, D. J., Wright, A. N., \& De Moortel, I. 2010, ApJ, 711, 990

Pascoe, D. J., Wright, A. N., \& De Moortel, I. 2011, ApJ, 731, 73

Roberts, B., Edwin, P. M., \& Benz, A. O. 1984, ApJ, 279, 857

Ruderman, M. S., \& Roberts, B. 2002, ApJ, 577, 475

Soler, R., Terradas, J., \& Goossens, M. 2011a, ApJ, 734, 80

Soler, R., Terradas, J., Verth, G., \& Goossens, M. 2011b, ApJ, 736, 10

Taroyan, Y., \& Erdélyi, R. 2009, Space Sci. Rev., 149, 229

Terradas, J., Arregui, I., Oliver, R., et al. 2008, ApJ, 679, 1611

Terradas, J., Goossens, M., \& Verth, G. 2010, A\&A, 524, A23

Tomczyk, S., \& McIntosh, S. W. 2009, ApJ, 697, 1384

Tomczyk, S., McIntosh, S. W., Keil, S. L., et al. 2007, Science, 317, 1192

Uchida, Y. 1970, PASJ, 22, 341

Verth, G., Terradas, J., \& Goossens, M. 2010, ApJ, 718, L102

Walsh, R. W., \& Ireland, J. 2003, A\&AR, 12, 1

Zacharias, P., Peter, H., \& Bingert, S. 2011, A\&A, 531, A97 